\title{
El Plan Andaluz de Sostenibilidad Energética 2007-2013 (PASENER)*
}

\author{
Antonio José Sánchez Sáez \\ Prof. Titular de Derecho Administrativo \\ Universidad de Sevilla
}

El pasado 13 de noviembre fue aprobado por el Consejo de Gobierno de la Junta de Andalucía el Plan Andaluz de Sostenibilidad Energética 2007-2013 (en adelante, PASENER), documento indicativo que forma parte del bloque de medidas que la Junta incluye en su Estrategia andaluza ante el Cambio Climático, adoptada en Acuerdo de 3 de septiembre de 2002 por el Consejo de Gobierno, y en la que se detallan hasta 140 medidas orientadas a reducir un $19 \%$ las emisiones de gases efecto invernadero en $2012^{1}$.

Recordemos que la Ley Orgánica 2/2007, de 19 de marzo, de reforma del Estatuto de Autonomía para Andalucía, establece como uno de los principios rectores de la CAA el impulso y desarrollo de las energías renovables, el ahorro y eficiencia energética (art. $37.1,21^{\circ}$ ), al tiempo que reconoce a Andalu-

\footnotetext{
${ }^{\circledR}$ La presente investigación se enmarca dentro del Proyecto "Régimen Jurídico de los Recursos Naturales (SEJ2007-66415/JURI)", con sede en el Área de Derecho Administrativo del Departamento de Derecho Administrativo, Derecho internacional Público y Relaciones internacionales de la Universidad de Sevilla, y financiado por el Ministerio de Educación y Ciencia.

${ }^{1}$ Las principales características que animan la creación de la Estrategia andaluza son la implicación explícita del Consejo de Gobierno (que se traduce en la creación de un presupuesto propio para dicha estrategia) y la voluntad de transmisión de los resultados obtenidos y de las propuestas aportadas a los órganos estatales en que participa la Junta de Andalucía. Las medidas que componen la Estrategia andaluza, aunque implican en realidad a toda la Junta de Andalucía, se han atribuido a una serie de Consejerías para que las desarrollen. Las Consejerías implicadas son las de Medio Ambiente, Innovación, Ciencia y Empresas, Agricultura y Pesca, Salud, Obras Públicas y Transportes y Educación. La Orden de 21 de noviembre de 2002 de la Consejería de Medio Ambiente creó el Panel de Seguimiento de la Estrategia Andaluza ante el Cambio Climático, dando cumplimiento así a una de las medidas que le fueron encomendadas en el Acuerdo de 3 de septiembre del Consejo de Gobierno, por el que se crea la Estrategia Andaluza ante el cambio climático.
} 
cía, como competencia compartida con el Estado, sobre las instalaciones de producción, distribución y transporte de energía, cuando este transporte transcurra íntegramente por el territorio de Andalucía y su aprovechamiento no afecte a otro territorio, sin perjuicio de sus competencias generales sobre industria. Asimismo le corresponde el otorgamiento de autorización de estas instalaciones, y el Fomento y gestión de las energías renovables y de la eficiencia energética, y la competencia, con respeto a las bases que establezca el Estado sobre la actuación económica general, de regulación de actividades de producción, depósito y transporte de energías, así como su autorización e inspección y control, estableciendo, en su caso, las normas de calidad de los servicios de suministro (art. 49). También reconoce el derecho de la CAA a participar en la designación de los miembros de la Comisión Nacional de Energía (art. 87) y ordena a los poderes públicos de la CAA la puesta en marcha estrategias dirigidas a evitar el cambio climático, potenciando las energías renovables y limpias, y llevando a cabo políticas que favorezcan la utilización sostenible de los recursos energéticos, la suficiencia energética y el ahorro (art. 204). Tan concienciada, en fin, se encuentra la Junta de Andalucía con las nuevas tecnologías propias de las energías renovables que se acabarán incluyendo como objeto de estudio en los planes educativos, conforme sugiere el art. 21 del nuevo Estatuto de Autonomía.

El Documento coincide también en su puesta de largo con la aprobación de la Estrategia Española de desarrollo sostenible, que se produjo el 23 de noviembre de 2007, dentro del cual tampoco falta una estrategia dedicada al cambio climático y energías limpias y del Plan Andaluz de Acción por el Clima: Programa de mitigación 2007-2013², y con el Informe que el Panel Intergubernamental sobre Cambio Climático (IPCC) presentó en Valencia el 17 de noviembre de 2007, en el que se advierte de manera inequívoca a los Gobiernos de la necesidad de "actuar con fuerza" antes de 2015 para salvar el clima

\footnotetext{
${ }^{2}$ Otros documentos que se han tenido en cuenta para su elaboración han sido el Código Técnico de la Edificación, aprobado por RD 314/2006, de 17 de marzo y el Real Decreto 661/2007, de 25 de mayo, por el que se regula la actividad de producción de energía eléctrica en régimen especial, que ha establecido un nuevo marco retributivo de las energías renovables. También, a un nivel más local, la creación de la Agencia Andaluza de la Energía por la Ley 4/2003, de 23 de septiembre, ha dotado a la Junta de un instrumento preciso dedicado al estudio y fomento de las energías renovables. En particular, es el organismo encargado de gestionar las ayudas e incentivos para la sostenibilidad energética en Andalucía (véase la Orden de 11 de abril de 2007, de la Consejería de Innovación, Ciencia y Empresa, que establece las bases reguladoras de un programa de incentivos para el desarrollo energético sostenible de Andalucía y efectúa su convocatoria para el año 2007).
} 
cuando se reúnan en Bali en diciembre para negociar la segunda fase del Protocolo de Kyoto ${ }^{3}$.

El PASENER tuvo como punto de partida el Acuerdo de 13 de junio de 2006, del Consejo de Gobierno de la Junta de Andalucía, por el que se aprobaba su formulación, y tiene como finalidad la creación de redes de dotaciones energéticas, completadas por fuentes de energías renovables en grado de suficiencia y autogestión en Andalucía ${ }^{4}$, que permita que toda la población de la Comunidad pueda contar un acceso de calidad a la energía independientemente del territorio en el que habiten. Sabemos que una de las principales virtualidades de las energías renovables, aparte de la consabida independencia económica de la importación del petróleo y de su proverbial respeto a la Naturaleza, es la "democratización" de los recursos energéticos, por cuanto que se hacen accesibles para cualquier persona que quiera instalarlas, en cualquier lugar, por aislado que esté.

Pero el PASENER no se queda ahí, sino que incorpora medidas de fomento de las energías renovables y actuaciones en materia de eficiencia energética (en realidad, el talón de Aquiles de los países occidentales, cuyo aumento de la demanda energética parece no tocar techo ${ }^{5}$ ), y el desarrollo de la tecnología asociada con estas fuentes de energía. Parte también de un análisis un tanto amargo en relación a la implantación real de las energías renovables, no tan rápida ni potente como se esperaba, y de datos poco sostenibles de la economía social, como la hiperurbanización del litoral, en donde se localizan buena parte del millón y medio de viviendas vacías que existen en la $\mathrm{CAA}^{6}$.

\footnotetext{
3 "Inequívoco" es también la expresión que usa el PASENER para referirse a la inexorabilidad del cambio climático. El Informe IPCC sitúa a España entre los países con más riesgos de ser castigados por el cambio climático, debido a los efectos que la desertificación producirá en el agua (reducción de las precipitaciones), pérdida de riqueza de los suelos, salinidad de los acuíferos, temperaturas extremas y aumento del nivel de las aguas del mar. En las páginas 11 y 12 del PASENER se concretan aún más los efectos para España del cambio climático, en una lista que pone los pelos de punta y que incluye problemas en el sector turístico, para la salud, la pesca, aumento de los incendios, etc.

${ }^{4}$ Sobre el concepto de "redes de energía", véase la página 23 y ss. del PASENER.

${ }^{5}$ Sobre el aumento del consumo de energía primaría en Andalucía, véase la pág. 29 del PASENER.

${ }^{6}$ El índice de motorización en inferior a la media nacional y muy inferior al de países como Alemania; se multiplican los puntos de extracción de agua ilegales, al tiempo que no llega a los niveles de suficiencia para el litoral; el porcentaje de energía primaria consumida procedente del petróleo no deja de aumentar, llegando ya al 50,4\%.
} 
La base de partida del PASENER es el Protocolo de Kyoto, y la técnica empleada es la de la planificación, en consonancia con las exigencias de la recién aprobada Ley 2/2007, de 27 de marzo, de fomento de las energías renovables y del ahorro y eficiencia energética de Andalucía, y al hilo de los anteriores Planes Energéticos de Andalucía (1995-2000 y 2003-2006), Estrategia andaluza contra el cambio climático y Estrategia andaluza de desarrollo sostenible: Agenda $21^{7}$.

El PASENER tiene la consideración de Plan con incidencia en la ordenación del territorio, conforme establece el nuevo apdo. 15ํㅡ del Anexo I (Planificación de infraestructuras energéticas) de la Ley 1/1994, de 11 de enero, de ordenación del territorio de la Comunidad Autónoma de Andalucía, tras la reforma sufrida por la citada Ley 2/2007, de 27 de marzo, de fomento de las energías renovables y del ahorro y eficiencia energética de Andalucía.

También tiene la consideración de Plan con efectos sobre el medio ambiente, a los efectos de la Ley 9/2006, de 28 de mayo, sobre Evaluación de los efectos de determinados planes y programas en el medio ambiente, lo que ha determinado que necesitara la elaboración de un Informe de sostenibilidad ambiental por parte del órgano promotor del mismo, esto es, la Consejería de Innovación, Ciencia y Empresa dirigido a identificar, describir y evaluar los probables efectos significativos sobre el medio ambiente que puedan derivarse de la aplicación del mismo, así como unas alternativas razonables, técnica y ambientalmente viables (entre las cuales la Ley baraja la posible alternativa cero, esto es, la no aprobación del Plan), que tengan en cuenta los objetivos y el ámbito territorial de aplicación del plan o programa(art. 8 de esa Ley). Conforme con su art. 10, además, tanto el PASENER como su Informe de sostenibilidad han tenido que ser puestos a disposición del público por un plazo de 45 días, que se configura más como un trámite de audiencia que de información pública, ya que las consultas sólo pueden ser realizadas por personas interesadas en el sentido estricto del término, esto es, conforme al art. 31 de la

${ }^{7}$ El PLEAN 2003-2006 ha sido desigualmente cumplido en sus objetivos de implantación de las distintas fuentes de energías renovables: se han cumplido los objetivos para la hidráulica especial, la solar fotovoltaica aislada, la solar fotovoltaica conectada o la biomasa de generación eléctrica, pero no así los de la eólica, atascada en un maremágnum de trámites burocráticos, en la dificultad para llegar a acuerdos con los Ayuntamientos, y en las escasas redes de evacuación, la solar térmica, la solar termoeléctrica, la biomasa térmica o los biocarburantes). Eso sí, gracias a las nuevas plantas de ciclo combinado de gas natural instaladas, Andalucía ha pasado de ser una Comunidad importadora de energía a ser autosuficiente, a pesar de algunos puntos negros, como Almería, que aún no está conectada a la red gasística. Vid. págs. 33 y 36 del PASENER. 
LRJAP y PAC. Igualmente, se somete a consulta de las Administraciones públicas afectadas y al público interesado, que dispondrán de un plazo mínimo de 45 días para examinarlo y formular observaciones ${ }^{8}$.

En fin, el PASENER incluye, en sus 194 págs. los siguientes contenidos:

1. El diagnóstico de la situación energética actual: infraestructuras de generación, transporte y distribución de la energía en Andalucía, del consumo de energía primaria y final: por clases de energía, sectores de actividad, y por unidades y estructuras territoriales; los ratios de consumo per cápita y del nivel de autoabastecimiento energético de la Comunidad Autónoma y análisis sobre los derechos de emisión de gases de efecto invernadero asignados a Andalucía para el sector energético.

2. La determinación de los objetivos generales a alcanzar, y la justificación de su coherencia con el modelo territorial regional, la planificación económica y la planificación ambiental de Andalucía. Se pretende llegar a un escenario de ahorro en el que los crecimientos acumulados de la demanda de energía total y primaria se cifrarían en el 21,3\% y 24,7\%, respectivamente, para 2014, con crecimientos medios anuales del 2,8 y 3,2, respectivamente. Los valores de energías renovables se exponen en la siguiente tabla, y en las págs. 91-94 del PASENER:

\begin{tabular}{|c|c|c|c|}
\hline Energian renowablet por tecnologint (parmmitrico) & 2007 & 2010 & 2013 \\
\hline Hidridulica rofoimen especiel & 129.0 & 137.6 & 140.0 \\
\hline Midräulice rógimen ordinumio & 464,2 & 476 & 476 \\
\hline Eolice & 1.204 & 4.000 & 4.000 \\
\hline Solar fotemeitaice & 36,2 & 55.4 & 100,0 \\
\hline Solar tormice & 407.000 & 765.220 & 2.242 .554 \\
\hline Soler termoeldetrica & 60 & 250 & 575 \\
\hline Biomats use térmico & 503,5 & 615.6 & 6.49 .0 \\
\hline Biomata generackibn tibctike & 169,9 & 209.9 & 256.0 \\
\hline Eiomase so-comburtidn & 0 & 61 & 122 \\
\hline Elogin uso térmico & 2,1 & 2,5 & 3.0 \\
\hline Biogis gener aciden eldectrice. & 16.0 & 17.2 & 20.1 \\
\hline Biocatburantes censums & 50 & 220 & 450 \\
\hline Diocarburantes peoducción & 263.7 & 2.000 & 2.300 \\
\hline $\begin{array}{l}\text { Energia prinsaria procederite de } \\
\text { fuentes menovables }\end{array}$ & 1.402 & 2.570 & 2.921 \\
\hline
\end{tabular}

${ }^{8}$ También podrán realizar consultas, dice la Ley, cualquier persona jurídica sin ánimo de lucro que cumpla los siguientes requisitos:

1. Que tenga como fines acreditados en sus estatutos, entre otros, la protección del medio ambiente en general o la de alguno de sus elementos en particular, y que tales fines puedan resultar afectados por el plan o programa de que se trate.

2. Que lleve al menos dos años legalmente constituida y venga ejerciendo de modo activo las actividades necesarias para alcanzar los fines previstos en sus estatutos. 
3. El análisis de la demanda de energía prevista en Andalucía hasta el año 2013 inclusive, de acuerdo con las previsiones de crecimiento de otros parámetros relacionados con el consumo energético, así como una previsión de dicha demanda hasta el año 2020.

4. La determinación y el análisis del potencial de las energías renovables de Andalucía.

5. La definición de los objetivos, criterios, directrices y principales intervenciones previstas relativas a:

- Las infraestructuras eléctricas, así como la calidad del suministro.

- Las infraestructuras gasistas, petrolíferas y sus derivados.

- Las infraestructuras necesarias para el fomento de las energías renovables.

- Actuaciones para el impulso del ahorro y la eficiencia energética.

6. La estructuración de las actuaciones y medidas en los siguientes Programas de implantación:

- Programa de fomento de energías renovables.

- Programa de ahorro y eficiencia energética.

- Programa de infraestructuras energéticas.

- Programa de innovación energética.

- Programa de difusión de la nueva cultura energética.

7. El análisis de los impactos del Plan sobre el empleo, la generación de valor añadido, las políticas de investigación y desarrollo, el medio ambiente, la salud pública y su incidencia en la ordenación del territorio.

8. La valoración económica de las actuaciones previstas.

9. Los instrumentos financieros y de gestión que deban ponerse en marcha por la Junta de Andalucía para la correcta aplicación del Plan.

10. La definición de los criterios para evaluación, seguimiento y revisión del Plan.

En definitiva, con el PASENER la Junta de Andalucía revisa de una manera más realista sus previsiones de desarrollo de las energías renovables, al tiempo que lanza un enorme arsenal de medidas (algunas ya existentes, otras 
por elaborar) que suponen un enfoque integral y sostenible del tema, insertándose, como ya plasmaba la Ley 2/2007, de 27 de marzo, de fomento de las energías renovables y del ahorro y eficiencia energética de Andalucía, en las políticas de transporte, urbanismo y ordenación del territorio.

Es un paso adelante en la buena dirección, pero está por ver, no obstante, que se lleven a cabo realmente y con la celeridad requerida las ambiciosas medidas de construcción de los puntos de conexión de las redes de distribución, la integración de las energías renovables en los edificios administrativos, la planificación del territorio de una manera que evite los desplazamientos y evite la construcción de nuevos asentamientos que demanden energía no disponible (con el obstáculo añadido de que ya se ha aprobado el POTA y muchos Planes de Ordenación del territorio de carácter subregional), o las medidas de simplificación administrativa de los procedimientos de licencia de las instalaciones respetando las medidas de garantía impuestas por el Real Decreto $661 / 2007$, de 25 de mayo, por el que se regula la actividad de producción de energía eléctrica en régimen especial.

Veamos ahora cuáles son las medidas más destacadas que prevé el Plan, sin duda su parte más importante por lo que tiene de prospectiva, que se integran dentro de cuatro programas, denominados "La Energía de los Ciudadanos", "Competitividad Energética", "Energía y Administración" e "Infraestructuras Energéticas". Incluimos en este resumen los comentarios literales que a ellas se hacen en el PASENER, con la indicación de que las demás medidas pueden consultarse en el Documento completo del Plan, que obra en la página web de la Consejería de Innovación, Ciencia y Empresa. Estos cuatro programas se dividen a su vez en varias líneas de actuación, que mencionamos también.

\section{PROGRAMA "LA ENERGÍA DE LOS CIUDADANOS"}

El cambio de modelo energético demanda el planteamiento de un esquema basado en el concepto social de la nueva cultura de la energía que, en forma análoga a la planteada en la planificación para la gestión de los recursos hídricos en forma de nueva "cultura del agua", sea capaz de dar respuesta a la necesidad de gestionar adecuadamente la demanda energética, evitando el despilfarro. En este nuevo modelo el ciudadano pasa a ser eje vertebrador, pero no tan solo por el hecho de asistir e incorporarse como agente pasivo al planteamiento de las medidas dispuesta por los órganos competentes en materia energética, sino como sujeto activo fundamental a la hora de participar 
en la toma de decisiones y en la difusión de un posicionamiento activo en la toma de conciencia sobre el problema de la energía.

Línea de actuación "Incorporación de la gestión eficiente de la energía e integración de las energías renovables en los hábitos de consumo"

- Programa de incentivos a la instalación de tecnologías renovables en el ámbito domestico particular y comunitario. Se continuará con la línea de incentivos establecidos para las instalaciones solares térmicas, la biomasa térmica y otras fuentes renovables. Se fomentara el uso de fuentes renovables para producción fundamentalmente de agua caliente sanitaria y calefacción. En el ámbito comunitario se pretende fomentar las redes de producción de agua caliente sanitaria y climatización (frío-calor) centralizada con energías renovables. Se fomentará, especialmente, la realización de instalaciones con fuentes renovables en zonas geográficas con escasa implantación en la actualidad.

- Promover la adecuación de las viviendas existentes a los requisitos energéticos incluidos en el certificado energético andaluz. La Ley 2/2007, de fomento de las energías renovables y del ahorro y eficiencia energética en Andalucía, establece la obligación a los edificios de nueva construcción de disponer de un certificado energético acreditativo del cumplimiento de los requisitos mínimos de eficiencia energética establecidos reglamentariamente. Con la presente medida se incentivaran actuaciones en ahorro y eficiencia energética en las viviendas existentes, tales como la mejora del aislamiento térmico, la mejora de la eficiencia energética de las instalaciones de calefacción y climatización o la mejora de la eficiencia energética de los sistemas de iluminación, como instrumento para adecuar su eficiencia energética a los requerimientos energéticos incluidos en el certificado

Energético andaluz.

- Plan de sustitución de electrodomésticos ineficientes. Se plantea facilitar a los ciudadanos el cambio de sus viejos electrodomésticos de baja eficiencia, y por tanto con mayores consumos energéticos, por electrodomésticos etiquetados energéticamente como clase A o superiores (A+ y A++). Adicionalmente se promoverá la realización de cursos de formación destinados a vendedores de electrodomésticos donde se les proporcionara formación adecuada para asesorar correctamente a sus potenciales usuarios sobre el etiquetado energético y las ventajas de los equipos catalogados con la categoría A y superiores $(\mathrm{A}+\mathrm{y} \mathrm{A}++)$. 
- Desarrollo de una línea de incentivos a la incorporación de vehículos turismos de mayor eficiencia energética. Los nuevos vehículos turismos permiten reducir el consumo energético y la contaminación ambiental al disponer de tecnologías más eficientes que la de los vehículos antiguos. Se incentivara la adquisición de vehículos turismos de propulsión eléctrica, híbrida o alimentados por gas natural, gases licuados del petróleo, hidrogeno o biocarburantes con alta concentración en la mezcla (B-100 y E-85), mediante ayudas económicas que palien el sobrémoste de estos vehículos alternativos.

\section{Línea de actuación "Información a los ciudadanos"}

- Difusión especifica dirigida a la ciudadanía sobre el Programa de Incentivos para el desarrollo energético sostenible en Andalucía. El fomento de un consumo energético consciente y del cambio de hábitos en el ciudadano pasa por el conocimiento y por hacerles participes de que para las inversiones en mejoras energéticas que se decidan acometer cuentan con el apoyo institucional de la Junta de Andalucía.

- Realizar campañas de fomento de buenas prácticas energéticas en el hogar, y promoción del consumo de productos y servicios de alta calificación energética. Con esta medida se pretende que el ciudadano participe y se involucre conscientemente en la necesidad de adoptar hábitos de control en su domicilio, tanto en lo referente al consumo energético directo, como a la reducción de la huella ecológica en cesta de la compra. La presente actuación se realizara en colaboración con las asociaciones vecinales, asociaciones de consumidores y centros educativos.

- Realizar campañas de fomento del uso de las energías renovables en el hogar. La medida contempla la realización de guías, campanas en medios de comunicación social, la organización y participación en jornadas, etc. Permitirá al consumido poder optar por fuentes renovables en el hogar con total garantía en cuanto a sus efectos y prestaciones.

- Promoción del certificado energético andaluz de viviendas. Se realizara una amplia campana de información al ciudadano sobre el certificado energético de viviendas regulado reglamentariamente por la Ley 2/2007 de fomento de las energías renovables y del ahorro y eficiencia energética de Andalucía, al objeto de explicar las ventajas energéticas de una vivienda calificada con A o At. Adicionalmente se creara una plataforma Web donde el ciudadano pueda cotejar sus consumos energéticos reales con los previstos en su certificado energético e identificar las causas de dicha desviación. 
- Difusión del etiquetado energético de vehículos turismos Mediante el Real Decreto 837/2002 se establece como obligatoria la colocación de una etiqueta sobre consumo de combustible y emisiones de CO2 de forma claramente visible en cada modelo de turismo nuevo. Complementariamente, con carácter voluntario, se establece la posibilidad de que la etiqueta incluya además la clasificación por consumo comparativo del coche. Con esta medida se pretende incidir en la capacidad de este etiquetado para adoptar criterios de ahorro y eficiencia energética en la compra de los vehículos.

\section{Línea de actuación "Formación de los ciudadanos"}

\section{PROGRAMA "COMPETITIVIDAD ENERGÉTICA"}

Las empresas andaluzas juegan un doble papel, como demandantes de energía y de tecnologías energéticas y como desarrolladoras y oferentes de dichas tecnologías. El consumo energético es crítico en el balance económico de las empresas. La actividad productiva es la primera en que se dejan sentir las alteraciones y medidas relacionadas con la energía, bien sea por la inestabilidad internacional del suministro energético, por la falta de adecuadas infraestructuras de distribución y abastecimiento, por la volatilidad de los precios de las fuentes energéticas de origen fósil, con los continuos incrementos en el precio del barril de petróleo, bien por ser quien recibe en primera instancia los efectos de las medidas de regulación y control de emisiones de gases de efecto invernadero.

Es evidente la necesidad de acometer cambios estructurales profundos que impliquen la incorporación de medidas con el objeto de incrementar el autoabastecimiento y la eficiencia en la transformación, transporte, distribución y uso final de la energía. Todo ello propiciara la formación y expansión de un conglomerado de empresas andaluzas de bienes y servicios, encaminadas a atender las nuevas demandas surgidas de la implementación del nuevo modelo energético. Por otra parte, en un mercado europeo unificado, el nivel de vanguardia en la competitividad lo establecen aquellas empresas que apuestan por la innovación, el ahorro y la eficiencia en los procesos productivos.

La elevada disponibilidad de recursos autóctonos de origen renovable plantea una oportunidad única para las empresas andaluzas de posicionarse tecnológicamente a la cabeza a nivel mundial, propiciando el óptimo aprovechamiento de las fuentes de energía limpias y exportando tecnologías y conocimientos a otras regiones del planeta. 


\section{Línea de actuación "Adopción de criterios de gestión eficiente e incorpora- ción de tecnologías renovables en la empresa"}

- Promover las inversiones en ahorro y eficiencia energética. Con esta medida se pretende impulsar las inversiones tendentes a la reducción del consumo energético de las diferentes instalaciones o equipos de los centros consumidores de energía de los diferentes sectores de actividad. De una forma mas precisa, se abarcaran aspectos tan diversos como la renovación de las instalaciones existentes de los edificios (calefacción, refrigeración, iluminación y producción de agua caliente sanitaria), rehabilitación de su envolvente térmica, mejora de los procesos e instalaciones industriales, etc.

- Promocionar la instalación de tecnologías renovables en las empresas. Con esta medida se pretende inducir en el sector productor de bienes y servicios la incorporación de tecnología basada en las fuentes de energía renovable, con especial incidencia en la mejora de los procesos y costes en la PYME.

- Promover el certificado energético andaluz en las instalaciones industriales. La Ley 2/2007, de fomento de las energías renovables y del ahorro y eficiencia energética de Andalucía, establece la obligación para los nuevos centros de consumo del sector industrial, a partir de un cierto nivel de consumo energético establecido reglamentariamente, de disponer de un certificado energético como documento acreditativo del cumplimiento de los requisitos energético exigidos reglamentariamente. Esta medida pretende promover en los nuevos centros industriales una alta calificación energética, mediante el desarrollo de campanas de comunicación y difusión de las ventajas energéticas de una alta calificación energética.

- Programa de fomento del ahorro energético y las energías renovables en el Sector turístico tradicional. El sector turístico tradicional representa uno de los sectores con mayor incidencia en el consumo energético del sector servicios en Andalucía. La importancia de este sector, unido al elevado potencial de ahorro energético y uso de las energías renovables existente en la actualidad en las instalaciones hoteleras andaluzas, justifica la necesidad de desarrollar un programa específico en materia energética para el sector turístico tradicional. En el citado programa se contempla el desarrollo de campanas de comunicación y difusión de las medidas de ahorro energético implementadles en las instalaciones hoteleras, ya sea de iluminación, climatización, lavandería o cocina, de las alternativas de uso las energías renovables, solar o biomasa, para la generación de agua caliente sanitaria o climatización de edificios o calentamiento/climatización de piscinas, y de los incentivos existentes para 
llevarlas a cabo. La presente medida se realizara en colaboración con las principales asociaciones empresariales de este sector.

- Fomentar la incorporación de sistemas de energías renovables como equipamiento de generación en zonas aisladas para el sector servicios, reforzando el concepto del turismo sostenible. Es necesario aumentar la confianza en el suministro energético mediante fuentes de energías renovables de zonas alejadas de núcleos urbanos, particularmente fomentando la viabilidad de soluciones energéticas aisladas de red en espacios naturales, alojamientos rurales, campamentos y áreas de acampada, cortijos, etc.

- Programa de fomento del ahorro energético y las energías renovables en la Industria agroalimentaria. La industria agroalimentaria en Andalucía representa aproximadamente el $70 \%$ del número total de industrias andaluzas y el $20 \%$ del consumo de energía del sector industrial. Se caracteriza por la gran variedad de procesos industriales, la elevada intensidad energética de algunos de sus subsectores y el elevado potencial de ahorro energético, superior al $13 \%$ del consumo global de energía primaria. En el citado programa se contempla el desarrollo de campanas de comunicación y difusión de las tecnologías de ahorro implementadles en este sector sustitución de derivados de petróleo por gas natural, aprovechamiento de purgas de calderas, instalación de recuperadores de calor, aprovechamiento de calores residuales, cogeneración), axial como las alternativas de uso de las energías renovables, fundamentalmente biomasa para la generación térmica, y la energía solar térmica a media temperatura para los procesos productivos. La presente medida se realizara en colaboración con las principales asociaciones empresariales de este sector con las que se podrán establecer acuerdos voluntarios.

- Fomentar los criterios de eficiencia energética y uso de energías renovables en el sector de la agricultura. Esta medida contempla el fomento de sistemas constructivos más eficientes energéticamente en la agricultura de invernaderos y el desarrollo de programas de modernización energética de los sistemas de regadío. Se promocionara la realización de auditorías energéticas en las actuales instalaciones de regadío. Se realizaran campanas de formación a agricultores en el uso eficiente de la energía (técnicas de cultivo, mejora energética de la maquinaria, uso eficiente del agua, etc.) y la oportunidad de las fuentes renovables para el sector.

- Promover el certificado energético andaluz en los edificios de nueva construcción. La ley 2/2007 de fomento de las energías renovables y del ahorro y eficiencia energética de Andalucía establece la obligación para los edifi- 
cios de nueva construcción de disponer de un certificado energético como documento acreditativo del cumplimiento de los requisitos energético exigidos reglamentariamente. Esta medida pretende promover en los edificios de nueva construcción una alta calificación energética, mediante el desarrollo de campanas de comunicación y difusión de las ventajas energéticas de un edificio calificado con A o A+.

- Promover la implementación de planes de gestión de la energía en los centros de consumo existentes. Los planes de gestión de la energía tienen por objeto asegurar en el tiempo el mantenimiento de la eficiencia energética de las instalaciones de un centro de consumo. Con la presente medida se pretende promover la implementación de planes de gestión de la energía en los centros de consumo existentes tanto del sector servicios como del sector industrial.

- Potenciar el desarrollo de la cogeneración en Andalucía. Esta medida contempla la identificación del potencial de cogeneración existente en Andalucía, la realización de estudios de viabilidad de nuevas instalaciones, axial como el establecimiento de un programa de incentivos al desarrollo de proyectos de cogeneración.

- Desarrollar un programa de renovación de la flota marítima andaluza. El ámbito del transporte de mercancías y de personas por vía marítima se ha dejado tradicionalmente fuera de las políticas de movilidad. Esta medida persigue incorporar en este sector criterios innovadores de eficiencia y de aplicación de energías renovables, como la sustitución de motores propulsores por otros de menor consumo de combustible, la mejora de los sistemas de propulsión o innovaciones en las operaciones de pesca que consuman menos combustible.

- Fomento de planes de movilidad en grandes centros industriales, comerciales o de servicios. La medida incluye el fomento de planes de transporte en grandes centros industriales, comerciales o de servicios y en las empresas de mas de 200 trabajadores, la asignación de un responsable para su gestión y el fomento de la realización de estudios energéticos a empresas de transporte industrial, de pasajeros o de mercancías para la mejora de la gestión energética de su flota. Esta medida se completa con el desarrollo de programas específicos de formación en conducción eficiente a conductores profesionales de vehículos turismos y vehículos industriales, programas específicos de formación de expertos en la gestión eficiente de combustible en flotas de transporte.

- Incrementar el número de biogasolineras en la Comunidad Autónoma Andaluza. 


\section{Línea de actuación "Difusión social de la incorporación de la nueva cultura energética al sistema productivo"}

- Fomento de la aplicación practica de las nuevas tecnologías energéticas fruto de la investigación andaluza y difusión de los resultados obtenidos. La Administración andaluza pretende servir de vector de aplicación y mediático de los logros obtenidos en $\mathrm{I}+\mathrm{D}+\mathrm{i}$ por las empresas y colectivos andaluces emergentes que, en el ámbito de la energía, destaquen generando valor añadido en aplicaciones tecnológicas y en la excelencia empresarial.

- Incorporar a los equipos e instalaciones de energías renovables un sello de Calidad Esta medida contempla la incorporación en Andalucía a los equipos e instalaciones de energías renovables un sello de calidad que asegure el proceso productivo de los componentes principales, el diseño de la instalación y su montaje. En una primera fase se establecerá la regulación que deberán cumplir los equipos e instalaciones, para en una segunda fase proceder a un proceso de certificación.

- Desarrollo de un marco legal que habilite el empleo de un sello de eficiencia energética a las empresas con una alta calificación energética. Esta medida contempla el desarrollo de un marco legal que permita a las empresas que acrediten en su certificado energético una elevada eficiencia energética el empleo en sus productos o campanas de comunicación de un sello de eficiencia energética. Los centros industriales existentes podrán acceder a este sello de eficiencia energética una vez acrediten su alta calificación energética mediante la obtención del correspondiente certificada energético andaluz.

\section{Línea de actuación "Fomento de la innovación e investigación en el ámbito energético"}

- Realizar estudios sectoriales con base para la identificación de las mejores tecnologías aplicables a cada subsector. La búsqueda permanente de identificación de barreras para cada diferente subsector del tejido empresarial andaluz, en lo relativo a la gestión eficiente de los consumos energéticos y a la innovación en los procesos productivos, requiere de un diagnostico previo a considerar con la aplicación de esta medida.

- Creación de redes entre universidades, centros de investigación y empresas andaluzas, favoreciendo el intercambio de conocimiento y la transferencia tecnológica Esta medida contempla la promoción de contratos y convenios de colaboración tecnológica entre empresas y centros de investigación y universidades andaluzas, el fomento de planes y programas de doctorado 
vinculados a empresas del sector energético y el desarrollo de un programa de intercambio de profesionales del sector energético andaluz con empresas de otras regiones del mundo.

\section{Línea de actuación "Apoyo al establecimiento de un tejido empresarial líder en tecnologías de energías renovables, ahorro y eficiencia energética"}

- Fomentar la colaboración de las entidades financieras en el desarrollo de proyectos energéticos mediante mecanismos de financiación por tercero. Se pretende con esta medida involucrar a las entidades bancarias en la financiación de proyectos energéticos mediante el desarrollo de instrumentos financieros especialmente adaptados a las necesidades de los mismos. Adicionalmente, dentro de la presente medida se elaborara un catalogo de instrumentos financieros de apoyo a las inversiones en ahorro energético para su difusión entre empresas del sector energético y entidades bancarias.

- Creación de una Red empresas energéticas. La red de empresas energéticas pretende ser un instrumento dinamizador del tejido empresarial andaluz en el sector de la energía, siendo el elemento a trabes del cual se pretende potenciar la integración de todas las empresas andaluzas del sector de la energía, fomentar la innovación, el desarrollo y la transferencia tecnológica desde los centros de investigación, facilitar la participación de las empresas andaluzas en mercados mas globalizados y fortalecer, en definitiva, la cadena de valor de cualquier industria o servicio energético andaluz.

\section{PROGRAMA "ENERGÍA Y ADMINISTRACIÓN"}

En la nueva cultura de la energía las administraciones publicas deben asumir el papel de auténticos catalizadores del cambio de paradigma, muy en particular las administraciones locales cuya labor en la implementación de medidas especificas es clave para la celeridad de la evolución hacia las estrategias basadas en modelos de consumo eficiente de los recursos y en el uso a gran escala de las energías renovables como fuentes de generación. Donde quizás se deba plasmar con mas fuerza el efecto promotor de las administraciones es en la necesidad de cohesionar e integrar todos los esfuerzos en políticas energéticas viables de buena gobernanza a todos los niveles, esto es, impulsando un autentico carácter transversal de la política energética.

Las políticas energéticas deben, por ejemplo, integrarse decisivamente al proceso de ordenación del territorio desde su inicio, introduciendo la di- 
mensión energética en las decisiones básicas de planificación y ordenación de usos para garantizar un desarrollo equilibrado y sostenible de las ciudades. No es admisible que el suministro energético sea una aportación posterior al estudio de ordenación del territorio, simplemente para satisfacer las demandas previstas.

Línea de actuación "Incorporación de la gestión eficiente de la energía e integración de las energías renovables en las administraciones"

- Desarrollo de la Red de Energía de la Junta de Andalucía. Entre las actuaciones a desarrollar por la Red de Energía en los centros de consumo adscritos a la Red destacan las siguientes: a) realización de estudios energéticos que permitan evaluar la viabilidad de las medidas de ahorro energético y uso de energías renovables implementadles en sus centros de consumo; b) asesoramiento y formación en la implementación de la normativa energética vigente en cada momento; c) asesoramiento en relación con las directrices generales y especificaciones técnicas de las contrataciones energéticas relevantes; d) seguimiento de las instalaciones energéticas para asegurar su eficiencia energética; e) interlocución con las compañías energéticas en las contrataciones de los suministros y equipamiento, y centralización de las incidencias que se reciban de estas compañías para facilitar y agilizar la resolución de las mismas; f) promoción de concursos públicos para la selección y contratación de servicios centralizados de suministro energético. La ejecución por la Red de los servicios descritos anteriormente requerirá el desarrollo de una serie de herramientas adecuadas de gestión (inventario, facturación, monitorización, incidencias) y de comunicación entre todos los actores implicados: responsables técnicos de la administración, Red, suministradores energéticos, etc. Para ello, esta previsto la construcción de una Red de Energía en Internet, a la que tendrán acceso todos las entidades publicas, concebida como herramienta mediante la cual se proporcione a los responsables técnicos de los centros de consumo los medios técnicos necesarios para optimizar el consumo energético de sus instalaciones, reducir el impacto ambiental, mejorar la calidad de los servicios públicos y ejecutar los Planes de Ahorro y Promoción de Energías Renovables de la Junta de Andalucía.

- Difusión de las líneas de apoyo a las Administraciones Locales en el ámbito de las mejoras energéticas que incorpora el programa de incentivos para el desarrollo energético sostenible de la Junta de Andalucía Resulta fundamental establecer un amplio programa de difusión por todo el territorio andaluz que actualice y ponga en conocimiento de las corporaciones locales el apoyo a las inversiones en proyectos de mejoras energéticas implementadles 
en las instalaciones municipales y la incorporación de fuentes renovables. Dado la importancia del alumbrado público como principal instalación de consumo energético en un municipio, se dedicara un especial esfuerzo a las medidas relacionadas con la renovación de instalaciones de alumbrado publico, la incorporación de elementos de regulación y control de estas instalaciones, o la incorporación de tecnología led’s.

- Impulsar la realización de Planes de Optimización Energética en los municipios andaluces. Los planes de optimización energéticos realizados hasta el 2006 en más de 200 municipios en Andalucía ponen de manifiesto el elevado potencial de ahorro energético existente actualmente. La medida incluye además del impulso a la realización de los planes de optimización energética, la promoción de la ejecución de las inversiones en ahorro y eficiencia energética en las instalaciones municipales, axial como el impulso a las energías renovables y a la diversificación de las fuentes de energía convencionales consumidas.

- Impulso a la incorporación de criterios de eficiencia energética en la Planificación territorial y urbanística. Esta medida favorece la incorporación de medidas de ahorro y eficiencia energética en la planificación del sistema de asentamientos y en el planeamiento urbanístico, a fin de incrementar la sostenibilidad y la calidad de los desarrollos urbanos.

- Fomentar la realización de planes de movilidad urbana. Redactar planes de movilidad de ámbito metropolitano y municipal: Impulsar la redacción de Planes de Movilidad Sostenible en los ámbitos territoriales gestionados por los consorcios metropolitanos de transporte y a nivel municipal, con el objetivo de asegurar la accesibilidad mediante transporte publico y medios no motorizados.

- Fomentar la realización de estudios energéticos de movilidad en las flotas de transporte público para la mejora de su gestión. Propuesta con la que se pretende evaluar el esquema de funcionamiento actual del transporte publico, localizando el potencial de mejora de la eficacia en las rutas realizadas y la gestión en general del mismo. Se contemplan además en esta medida el desarrollo de experiencias pilotos para la mejora del transporte público y su integración con otros modos de transporte.

- Desarrollar un programa de renovación de la flota de vehículos de transporte público urbano. Renovación de la flota de vehículos de transporte publico urbano de ayuntamientos o empresas publicas con responsabilidad en 
el trasporte publico urbano, alimentados con gasolina o asoleo por vehículos de propulsión híbrida, con pilas de combustible, impulsados a gas natural o biocarburantes. También se contempla en esta medida la incorporación de Tic para la mejora de la gestión de flotas de transporte público.

- Desarrollar de un modelo de Ordenanza municipal de Ahorro y Eficiencia Energética y uso de las energías renovables de aplicación en los municipios andaluces. La adopción de esta medida supondría la articulación de un marco normativo municipal común en Andalucía que establezca criterios mínimos de ahorro y eficiencia energética, axial como de la inclusión de instalaciones de energía renovables. El desarrollo de la presente medida se realizara en colaboración con la Federación Andaluza de Municipios (FAMP).

- Fomentar los contratos de "servicios energéticos" en la explotación de las instalaciones municipales. Con esta medida se pretende la mejora de la eficacia y gestión energética de las instalaciones municipales, mediante la implementación de técnicas de gestión energética adecuadas para el control de sus consumos energéticos.

- Incorporar criterios de eficiencia energética en las compras públicas o en la selección de empresas concesionarias de un servicio público. Se pretende con esta medida incorporar los criterios de eficiencia energética la selección de cualquier producto o servicio que se quiera contratar desde cualquier administración publica.

- Incluir el criterio de eficiencia energética en el equipamiento con consumo eléctrico del Catalogo de Bienes Homologados de adquisición por la Junta de Andalucía La Administración Publica Andaluza pretende asumir criterios de responsabilidad social corporativa prestando especial atención a la adquisición consciente de bienes de equipo de elevada eficiencia energética.

- Fomentar la figura del gestor energético municipal y la creación de una red de gestores energéticos municipales. Se pretende promover la figura del asesor o consultor técnico en materia de energía que asesoren de manera activa y transversal en la toma de decisiones en las administraciones locales y en la implementación de las ordenanzas y planes de optimización energética municipales.

\section{Línea de actuación "Dinamización del sector energético a través de la admi- nistración"}

- Desarrollo Reglamentario de la Ley de fomento de las Energías Renovables y del Ahorro Energético. Esta medida pretende propiciar el desarrollo 
de esta Ley en estrategias, objetivos y medidas de apoyo específicos en el menor tiempo posible. El reglamento, en una primera fase regulara: a) La obligación del uso de las energías renovables en edificios de la Junta de Andalucía. b) La obligación del uso de los biocarburantes en los autobuses de transporte publico que presten servicio regular de viajeros. c) El aprovechamiento energético del biogás. d) Establecimiento y regulación del Certificado Energético Andaluz para nuevos edificios y centros industriales.

- Desarrollo de normativa técnica de aplicación a las instalaciones de energías renovables en Andalucía. Esta medida pretende propiciar, mejorar y asegurar las prestaciones y funcionamiento de las instalaciones de energías renovables existente en Andalucía. La normativa desarrollada será de aplicación a las instalaciones realizadas en Andalucía, deberá adecuarse a la nacional y europea, particularizando para nuestra Comunidad al objeto que de respuesta al funcionamiento de las instalaciones debido a nuestra climatología, topología de edificios, uso y tipo de instalaciones, etc.

- Elaborar un procedimiento administrativo específico para la instalación de Energía solar fotovoltaica en Andalucía. Se pretende con este procedimiento establecer una norma para la autorización, aprobación de proyecto, declaración de utilidad publica, construcción, puesta en servicio, modificación, transmisión y cierre de instalaciones fotovoltaicas aisladas y conectadas a red, axial como su inclusión en el Registro de instalaciones de producción en régimen especial.

- Desarrollo de procedimiento administrativo mediante Tic para la agilización de los expedientes de autorización de las infraestructuras asociadas a tecnologías renovables y de alta eficiencia energética. Se pretende generar y sistematizar herramientas para la tramitación de expedientes relacionados con el ahorro, la eficiencia energética y las energías renovables, en la administración andaluza.

- Elaboración de un Plan de identificación de Áreas Preferentes de Energías Renovables (APER). La Ley 2/2007 de fomento de las energías renovables y del ahorro y la eficiencia energética de Andalucía define las Áreas Preferentes de Energías Renovables (APER) como posibles zonas compatibles para infraestructuras de generación y transformación de energías renovables. Con esta medida se pretende identificar dichas zonas y analizar los recursos renovables y la viabilidad de sus aprovechamientos.

- Realizar un programa de promoción de los cultivos energéticos. La medida contempla la realización de un programa de promoción de los cultivos 
energéticos, con la participación del sector público y privado, que propicie el desarrollo de la agra energía en Andalucía.

- Realizar un programa de biocarburantes. La medida contempla la realización de un programa de biocarburantes que analice las materias primas, las necesidades de $\mathrm{I}+\mathrm{D}+\mathrm{i}$, los sistemas de producción y los usos finales. Contribuir desde la planificación territorial y urbanística a reducir las necesidades de movilidad, fomentar el transporte público, las redes de transporte no motorizado y optimizar el diseño de urbanizaciones y edificaciones para mejorar la eficiencia energética. Se trata de conseguir con esta medida que se corrijan en origen desequilibrios estructurales en la ordenación del territorio y urbanística que causan, con posterioridad, situaciones de demanda energética (residencial y transporte) incompatibles con los objetivos de este Plan y los del Plan de Ordenación del Territorio de Andalucía.

- Introducir la evaluación de los consumos energéticos durante el ciclo de vida de los sectores urbanizables en estudio, como criterio de dimensionamiento del planeamiento urbanístico. En el proceso de diseño y dimensionamiento de la ciudad debe incorporarse la dimensión energética, con objeto de valorar la viabilidad del ciclo integral (incorporando recursos básicos y emisiones) de un crecimiento urbanístico municipal y su compatibilidad con los objetivos del PASENER y del Plan de Ordenación del Territorio de Andalucía. Se deberán fijar, en este sentido, módulos solventes de estimación por unidades físicas y criterios contrastables en la simulación para establecer los límites que sean precisos en su caso por parte de la administración urbanística y territorial.

- Contribuir eficazmente a que en procesos territoriales y urbanísticos se prevean las reservas y ordenaciones de usos necesarias para atender a las necesidades de las redes e infraestructuras energéticas. Se trata con esta medida de mejorar los procedimientos de planificación territorial y municipal para que se integren las necesidades de reserva de suelos y adecuada articulación de usos en la planificación de las redes energéticas y sus infraestructuras asociadas.

Línea de actuación "Acercamiento a la Sociedad del Conocimiento a través de la investigación e innovación en el ámbito energético"

\section{PROGRAMA "INFRAESTRUCTURAS ENERGÉTICAS"}

La ordenación territorial y la operación de las infraestructuras de transformación, transporte y distribución de la energía, deben evolucionar, desde 
un modelo basado en grandes centros de producción, hacia un nuevo modelo energético de generación local y consumo in situ, en el que participen, de forma creciente, las energías renovables. La garantía de suministro y el establecimiento de un sistema de infraestructuras de transformación, transporte y distribución de energía eficiente y respetuosa con el medio ambiente son los pilares de este programa.

Línea de actuación "Garantizar un suministro energético de calidad a todos los andaluces y promocionar un sistema de infraestructuras eficiente, limpio distribuido en toda Andalucía, que impulse el desarrollo económico manteniendo en todo momento como premisa el respeto y conservación del medio ambiente"

- Regular normativamente protección de la calidad del suministro eléctrico de los andaluces. Se pretende con esta medida recopilar en un Decreto la base reguladora necesaria para mejorar la calidad del suministro eléctrico en Andalucía, desarrollar el régimen jurídico de las acometidas eléctricas y regular los derechos y obligaciones de los usuarios del servicio eléctrico.

- Dotar de gas natural canalizado a los núcleos urbanos situados en el litoral y en los centros regionales. Llevar la red de gas natural a las capitales de Cádiz y Almería así como extender la infraestructura de distribución de gas natural a toda la banda litoral y los centros regionales, considerándola una infraestructura básica para su desarrollo socioeconómico.

- Realización de un Programa de acercamiento de las infraestructuras de gas natural zonas productivas de Andalucía. En el marco de este Programa se analizara la viabilidad de llevar el gas natural a localizaciones en las que se ubiquen sectores productivos con elevadas demandas energéticas.

Línea de actuación "Conocimiento para la gestión eficiente del sistema de infraestructuras"

- Desarrollo de un sistema de información no-líen de la generación de energía eléctrica en régimen especial en Andalucía. Esta medida tiene como objetivo conocer en tiempo real la cobertura de la demanda eléctrica de Andalucía con fuentes renovables y tecnologías eficientes acogidas al régimen especial.

- Impulsar la constitución en Andalucía de centros de control de generación en régimen especial. Se pretende con esta medida atraer la instalación de centros de control de la producción acogida al régimen especial de generación a los que obliga el Real Decreto 661/2007. 